\title{
THE SUN GRAPH IS DETERMINED BY ITS SIGNLESS LAPLACIAN SPECTRUM*
}

\author{
M. MIRZAKHAH ${ }^{\dagger}$ AND D. KIANI ${ }^{\dagger \ddagger}$
}

\begin{abstract}
For a simple undirected graph $\mathrm{G}$, the corresponding signless Laplacian matrix is defined as $D(G)+A(G)$ in which $D(G)$ and $A(G)$ are degree matrix and adjacency matrix of $G$, respectively. The graph $\mathrm{G}$ is said to be determined by its signless Laplacian spectrum, if any graph having the same signless Laplacian spectrum as $G$ is isomorphic to $G$. Also the Sun graph of order $2 n$ is a cycle $C_{n}$ with an edge terminating in a pendent vertex attached to each vertex. Among other things, one result in this paper is that the Sun graphs are determined by their signless Laplacian spectrum.
\end{abstract}

Key words. Sun graph, Signless Laplacian matrix, Cospectral graphs.

AMS subject classifications. 05C50, 05C90.

1. Introduction. Let $G$ be a simple undirected graph with $n$ vertices and $m$ edges. Let $V(G)$ and $E(G)$ denote the vertex set and edge set of $G$, respectively. Also let $A(G)$ be the $(0,1)$-adjacency matrix of $G$. Since $A(G)$ is a symmetric matrix, its eigenvalues are real numbers. The eigenvalues of $A(G)$ are denoted by $\lambda_{1}(G), \ldots, \lambda_{n}(G)$, where we take them to be arranged in non-increasing order. Let $I(G)$ be the (vertex-edge) incidence matrix of $G$. Also the identity matrix of order $n$ will be denoted by $I_{n}$.

Recall that the Laplacian matrix and the signless Laplacian matrix of $G$ are defined as $L(G)=D(G)-A(G)$ and $Q(G)=D(G)+A(G)$, respectively, where $D(G)$ is the diagonal matrix whose diagonal entries are the vertex degrees of $G$. As it is well-known, the matrices $L(G)$ and $Q(G)$ are positive semi-definite and they have the same characteristic polynomial if and only if $G$ is a bipartite graph. The eigenvalues of the matrices $L(G)$ and $Q(G)$, are denoted by $\mu_{1}(G) \geq \mu_{2}(G) \geq \ldots \geq$ $\mu_{n}(G)=0$ and $\nu_{1}(G) \geq \nu_{2}(G) \geq \ldots \geq \nu_{n}(G)$, respectively. Furthermore, the second smallest Laplacian eigenvalue of $G, \mu_{n-1}(G)$, is called the algebraic connectivity, and

*Received by the editors March 5, 2010. Accepted for publication on July 31, 2010. Handling Editor: Stephen Kirkland.

$\dagger$ Department of Pure Mathematics, Faculty of Mathematics and Computer Science, Amirkabir University of Technology (Tehran Polytechnic), P.O. Box 15875-4413, Tehran, Iran (mirzakhah@aut.ac.ir,dkiani@aut.ac.ir).

${ }^{\ddagger}$ School of Mathematics, Institute for Research in Fundamental Sciences (IPM), P.O. Box 193955746, Tehran, Iran. Supported in part by IPM Grant No. 89050116 and National Foundation of Elites. 
$\mu_{n-1}(G)=0$ if and only if the graph $G$ is disconnected (see $[6,9]$ ).

Suppose that $M(G)$ is a matrix associated with the graph $G$. The spectrum of $M(G)$ is the multiset containing its eigenvalues, each with its multiplicities, and we usually write the multiplicities as exponents (i.e. $\lambda_{1}^{m_{1}}, \ldots, \lambda_{r}^{m_{r}}$, where $m_{i}$ is the multiplicity of the eigenvalue $\lambda_{i}$ for $i=1, \ldots, r$ ). Two graphs $G$ and $H$ are said to be $M$-cospectral if they have equal $M$-spectrum, that is spectrum of $M(G)$ is equal to the spectrum of $M(H)$. In addition, the graph $\mathrm{G}$ is said to be determined by its $M$-spectrum, if any graph having the same $M$-spectrum as $G$ is isomorphic to $G$.

Let $P_{n}, C_{n}$, and $\mathcal{L}(G)$ be the path, the cycle on $n$ vertices, and the line graph of the graph $G$, respectively. If $e \in E(G)$, then $G-e$ denotes the subgraph of $G$ with vertex set $V(G)$ and edge set $E(G) \backslash\{e\}$. Also if $v \in V(G)$, then the graph $G-v$ is an induced subgraph of $G$ and obtained from $G$ by deleting the vertex $v$ and all edges incident with it.

A bicyclic graph is a connected graph in which $m=n+1$. Two paths are internally disjoint, if they do not have any vertex in common, except the first and the last ones. The graph $\theta_{p, q, r}$ is the union of three internally disjoint paths $P_{p}, P_{q+2}$, and $P_{r+2}$ which have the same two distinct end vertices, where $p \geq 2, q \geq 0$ and $r \geq 0$. The graph $\beta_{p, q}$ is the union of two cycles $C_{p}$ and $C_{q}$ with precisely one vertex in common, where $p \geq 3$ and $q \geq 3$. Also the dumbbell graph, $\gamma_{p, q, r}$, consists of two vertex disjoint cycles $C_{p}$ and $C_{q}$ joined by a path $P_{r}$ having only its end vertices in common with the cycles, where $p \geq 3, q \geq 3$ and $r \geq 2$.

Connected graphs in which the number of edges equals the number of vertices are called unicyclic graphs. Therefore, a unicyclic graph is either a cycle or a cycle with trees attached. A unicyclic graph containing an odd cycle (even cycle) is called odd unicyclic (even unicyclic). A vertex of degree one is called a pendent vertex. Furthermore, the Sun graph of order $2 n$ is a cycle $C_{n}$ with an edge terminating in a pendent vertex attached to each vertex, and it is denoted by $S G_{n}$ (see Fig. 1).

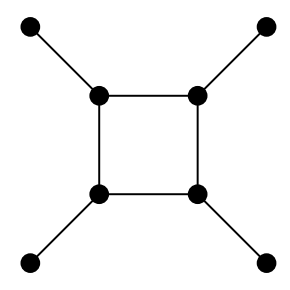

Fig. 1. $S G_{4}$

Which graphs are determined by their spectrum, seems to be a difficult problem in algebraic graph theory. Recently, this problem has attracted some research attention. 
For background and some known results on this problem, we refer the reader to $[1,11,7,10]$ and the references therein. In [1] it is proved that the Sun graph is determined by its Laplacian spectrum, and an odd Sun graph is determined by its adjacency spectrum. In particular, it is shown that for even $n$, there exist values of $n$ for which the Sun graph is not determined by its adjacency spectrum $(n=4$ and 16). Here, we prove that the Sun graph is determined by its signless Laplacian spectrum.

2. Basic Properties. In this section, we relate some useful results about signless Laplacian spectrum, which are essential tools for the next section.

The subdivision graph $S(G)$ of the graph $G$, obtained by inserting an additional vertex into each edge of $G$. Evidently, its adjacency matrix is of the form

$$
\left[\begin{array}{cc}
0 & I(G) \\
I(G)^{t} & 0
\end{array}\right]
$$

where $I(G)$ is the incidence matrix of $G$ (see e.g. [2]).

LEMMA 2.1. [8] The adjacency eigenvalues of the graph $S(G)$ are in the form of $\pm \sqrt{\nu_{i}(G)}$.

ExAmple 2.2. Applying Lemma 2.1 to the graph $K_{3}$, we see that the adjacency spectrum of the graph $S\left(K_{3}\right)=C_{6}$ is in the form of $(-2)^{1},(-1)^{2}, 1^{2}, 2^{1}$.

Suppose $G$ and $H$ are two graphs with disjoint vertex sets of orders $n_{1}$ and $n_{2}$, respectively. The product of $G$ and $H$, say $G \times H$, is a graph with vertex set $V(G) \times V(H)$ and two vertices $\left(v_{1}, u_{1}\right) \in V(G \times H)$ and $\left(v_{2}, u_{2}\right) \in V(G \times H)$ are adjacent if and only if $v_{1} v_{2} \in E(G)$ and $u_{1} u_{2} \in E(H)$. It is easy to see that, the adjacency eigenvalues of the graph $G \times H$ are $\lambda_{i}(G) \lambda_{j}(H)$, where $i=1, \ldots, n_{1}$; and $j=1, \ldots, n_{2} ;$ (see e.g. [2]).

Using the above statements, we have the following theorem.

TheOREm 2.3. Let $G$ be a graph. Then $\prod_{i=1}^{n} \nu_{i}(G)^{r_{i}}$ is the adjacency eigenvalue of the product of $r$ disjoint copies of the graph $S(G)$, where $r=2 \sum_{i=1}^{n} r_{i}, r>0$ and $r_{i}$ are non-negative integers, for $i=1, \ldots, n$.

Let $G$ be a connected graph. Also assume that the characteristic polynomial of the signless Laplacian matrix of $G$ is denoted by

$$
Q_{G}(x)=\sum_{i=0}^{n}(-1)^{i} \zeta_{i} x^{n-i} .
$$

Using the terminology and notation from [4], a spanning subgraph of $G$ whose connected components are trees or odd unicyclic graphs is called a $T U$-subgraph of $G$. Suppose that a $T U$-subgraph $H$ of $G$ contains $c$ odd unicyclic graphs and $s$ trees 
such as $T_{1}, \ldots, T_{s}$. Then the weight $W(H)$ of $H$ is defined by $W(H)=4^{c} \prod_{i=1}^{s} n_{i}$, in which $n_{i}$ is the number of the vertices of $T_{i}$. Note that if $H$ contains no tree, then $W(H)=4^{c}$. According to the following theorem, the $Q$-coefficients $\zeta_{i}$ can be expressed in terms of the weights of $T U$-subgraphs of $G$.

Theorem 2.4. [4] For $\zeta_{i}$ as above, we have $\zeta_{0}=1$ and

$$
\zeta_{i}=\sum_{H_{i}} W\left(H_{i}\right), \quad i=1, \ldots, n,
$$

where the summation runs over all TU-subgraphs $H_{i}$ of $G$ with $i$ edges.

REMARK 2.5. In Theorem 2.4, the $T U$-subgraph $H_{i}$ with $i$ edges, contains $n-i$ trees. Therefore all connected components of $H_{n}$ are odd unicyclic, in which $H_{n}$ is the $T U$-subgraph of $G$ with $n$ edges.

Lemma 2.6. Suppose $G$ is a graph and $G^{\prime}$ is a spanning subgraph of $G$, then $\operatorname{det}\left(Q\left(G^{\prime}\right)\right) \leq \operatorname{det}(Q(G))$. Moreover, if $v$ is a pendent vertex of $G$, then $\operatorname{det}(Q(G))=$ $\operatorname{det}(Q(G-v))$.

Proof. Let $X \subseteq E(G)$ and $G^{\prime}=G-X$. We denote the set of all $T U$-subgraphs of $G$ with $n$ edges by $\mathcal{F}(G)$. Therefore, $\emptyset \subseteq \mathcal{F}\left(G^{\prime}\right) \subseteq \mathcal{F}(G)$. So by Theorem 2.4 we have

$$
\operatorname{det}\left(Q\left(G^{\prime}\right)\right)=\sum_{H_{n} \in \mathcal{F}\left(\mathcal{G}^{\prime}\right)} W\left(H_{n}\right) \leq \sum_{H_{n} \in \mathcal{F}(G)} W\left(H_{n}\right)=\operatorname{det}(Q(G)) .
$$

Furthermore, let $v$ be a pendent vertex of $G$. We have $W\left(H_{n}-v\right)=W\left(H_{n}\right)$, where $H_{n}$ is a $T U$-subgraph of $G$ with $n$ edges. Therefore by Theorem 2.4 and Remark 2.5, $\operatorname{det}(Q(G))=\operatorname{det}(Q(G-v))$.

TheOREM 2.7. Let $G$ be a graph with $n$ vertices and $m$ edges;

(i) $\operatorname{det}(Q(G))=0$ if and only if $G$ has at least one bipartite connected component.

(ii) $\operatorname{det}(Q(G))=4$ if and only if $G$ is an odd unicyclic graph.

(iii) Suppose $G$ is a non-bipartite connected graph and $m>n$. Then $\operatorname{det}(Q(G)) \geq$ 16 and equality holds if and only if $G$ is a non-bipartite bicyclic graph with $C_{4}$ as its induced subgraph.

Proof. (i) can be proved by Corollary 2.2 of [4].

(ii) For the sufficiency part, let $G$ be an odd unicyclic graph. Hence, Theorem 2.4 yields $\operatorname{det}(Q(G))=4$. For the necessity part, suppose that $\operatorname{det}(Q(G))=4$. By $(i)$, all connected components of $G$ are non-bipartite. Assume that $G$ is not an odd unicyclic graph, then we have $m=n+k$, where $k \geq 1$. By induction on $k$, we prove that 
$\operatorname{det}(Q(G)) \geq 16$. If $k=1$, then $G$ is a bicyclic graph. Since $G$ is non-bipartite, without loss of generality, by Lemma 2.6, we may assume that one of the following cases occurs:

Case 1. $G \cong \theta_{p, q, r}$ such that $p+q$ and $p+r$ are odd and even numbers, respectively. So in order to obtain $T U$-subgraphs of $G$ with $n$ edges, we should delete one edge from the even cycle $C_{p+r}$. Clearly, the length of this cycle is at least four. Then by Theorem 2.4, we have $\operatorname{det}\left(Q\left(\theta_{p, q, r}\right)\right) \geq 16$ and equality holds if $p+r=4$.

Case 2. $G \cong \beta_{p, q}$ such that $p$ is an odd number. If $q$ is an odd number, then we have $\operatorname{det}\left(Q\left(\beta_{p, q}\right)\right)=4(p+q)$, otherwise $\operatorname{det}\left(Q\left(\beta_{p, q}\right)\right)=4 q$. It implies that $\operatorname{det}\left(Q\left(\beta_{p, q}\right)\right) \geq 16$ and equality holds if $q=4$.

Case 3. $G \cong \gamma_{p, q, r}$, this case is similar to the Case 2 .

Now suppose that $k \geq 2$. Then, there exists at least one cycle $\mathcal{C}$ such that by removing each edge of it, the remaining graph is a connected non-bipartite graph with at least $n+1$ edges. But we know that

$$
\operatorname{det}(Q(G)) \geq \sum_{e \in \mathcal{C}} \operatorname{det}(Q(G-e)) \geq 48 .
$$

This completes the proof of the $(i i)$ and (iii).

Lemma 2.8. Suppose $u, v$ are two non-adjacent vertices in the graph $G$ which have the same neighbors $(\operatorname{deg}(u)=\operatorname{deg}(v)=r)$. Then $G$ has $r$ as its $Q$-eigenvalue.

Proof. The proof of this lemma easily follows from $\operatorname{det}(r I-Q(G))=0$.

Lemma 2.9. Let $G$ be a graph with $t(G)$ triangles and $n_{i}$ vertices of degree $i$. Then

$$
t(\mathcal{L}(G))=t(G)+\sum_{i=3}^{\Delta(G)}\left(\begin{array}{c}
i \\
3
\end{array}\right) n_{i}
$$

where $\mathcal{L}(G)$ is the line graph of $G$ and $\Delta(G)$ is the maximum degree of a vertex of $G$.

TheOREm 2.10. [5, Lemma 2] Let $G$ be a graph on $n$ vertices and $m$ edges. Suppose that $e \in E(G)$ and $G^{\prime}=G-e$. Then $\nu_{1}(G)>\nu_{1}\left(G^{\prime}\right) \geq \nu_{2}(G) \geq \nu_{2}\left(G^{\prime}\right) \geq$ $\ldots \geq \nu_{n}(G) \geq \nu_{n}\left(G^{\prime}\right)$.

TheOREm 2.11. [4, Corollary 2.2] In any graph the multiplicity of the eigenvalue 0 of the signless Laplacian matrix is equal to the number of bipartite components.

Theorem 2.12. [4, Proposition 2.5] If two graphs are Q-cospectral, then their line graphs are cospectral with respect to the adjacency matrix. 
Theorem 2.13. [4, Corollary 4.3] Let $G$ be a graph with $n$ vertices, $m$ edges, $t$ triangles and vertex degrees $d_{1}, d_{2}, \ldots, d_{n}$. Let $T_{k}=\sum_{i=1}^{n} \nu_{i}^{k},(k=0,1,2, \ldots)$ be the $k$ th spectral moment for the $Q-$ spectrum. Then we have

$$
T_{0}=n, \quad T_{1}=\sum_{i=1}^{n} d_{i}=2 m, \quad T_{2}=2 m+\sum_{i=1}^{n} d_{i}^{2}, \quad T_{3}=6 t+3 \sum_{i=1}^{n} d_{i}^{2}+\sum_{i=1}^{n} d_{i}^{3} .
$$

TheOrem 2.14. [3, Section 5] If $G$ is a connected graph of order $n \geq 4$ and maximum degree $\Delta(G)$, then $\nu_{1}(G) \geq 1+\Delta(G)$. Also equality holds if and only if $G$ is the star graph.

3. The Sun Graph. In this section, we show that the Sun graph is determined by its $Q$-spectrum.

Lemma 3.1. The matrix $Q\left(S G_{n}\right)$ has $2+\lambda_{i} \pm \sqrt{1+\left(\lambda_{i}+1\right)^{2}}$ as its eigenvalues, where $\lambda_{i}=\cos \frac{2 \pi i}{n}$, for $i=1, \ldots, n$.

Proof. By a suitable labelling of vertices of $S G_{n}$, we may assume that

$$
Q\left(S G_{n}\right)=\left[\begin{array}{cc}
3 I_{n}+A\left(C_{n}\right) & I_{n} \\
I_{n} & I_{n}
\end{array}\right] .
$$

Therefore $Q_{S G_{n}}(x)=\operatorname{det}\left(x I_{2 n}-Q\left(S G_{n}\right)\right)=(x-1)^{n} A_{C_{n}}\left(x-3-\frac{1}{x-1}\right)$, in which $Q_{S G_{n}}(x)$ and $A_{C_{n}}(x)$ are characteristic polynomials of matrices $Q\left(S G_{n}\right)$ and $A\left(C_{n}\right)$, respectively. Notice that $A_{C_{n}}\left(x-3-\frac{1}{x-1}\right)$ is not a polynomial. One may see that, $Q\left(S G_{n}\right)$ does not have 1 as its eigenvalue, because the rows of the matrix

$$
I-Q\left(S G_{n}\right)=\left[\begin{array}{cc}
-2 I_{n}-A\left(C_{n}\right) & -I_{n} \\
-I_{n} & 0
\end{array}\right],
$$

are linearly independent, therefore $\operatorname{det}\left(I-Q\left(S G_{n}\right)\right) \neq 0$. Hence, $x-3-\frac{1}{x-1}$ is an eigenvalue of the matrix $A\left(C_{n}\right)$, if $x$ is a root of $Q_{S G_{n}}(x)$. Therefore, the $Q$-spectrum of $S G_{n}$ is in the form of $2+\lambda_{i} \pm \sqrt{1+\left(\lambda_{i}+1\right)^{2}}$, where $\lambda_{i}=\cos \frac{2 \pi i}{n}(i=1, \ldots, n)$.

Corollary 3.2. The matrix $Q\left(S G_{n}\right)$ does not have 1 as its eigenvalue.

COROLlary 3.3. The largest eigenvalue of the matrix $Q\left(S G_{n}\right)$ is equal to $3+\sqrt{5}$, for every positive integer $n \geq 3$.

Now, suppose $\mathcal{G}$ is non-isomorphic $Q$-cospectral with $S G_{n}$. By Theorem 2.13, $\mathcal{G}$ has the same number of vertices and edges as $S G_{n}$. Furthermore, Theorem 2.14 and Corollary 3.3 imply that $\Delta(\mathcal{G}) \leq 4$. Suppose $\mathcal{G}$ has $n_{i}$ vertices of degree $i$, for $i=0,1,2,3,4$.

TheOREm 3.4. Suppose $\mathcal{G}$ is a graph without any isolated vertex and is $Q_{-}$ cospectral with $S G_{n}(n \geq 4)$. Then $\mathcal{G}$ must be isomorphic to $S G_{n}$. 
Proof. Corollary 4.3 of [4] implies that

$$
\sum_{i=1}^{4} n_{i}=2 n, \quad \sum_{i=1}^{4} i n_{i}=\sum_{i=1}^{n} d_{i}=4 n, \quad \sum_{i=1}^{4} i^{2} n_{i}=\sum_{i=1}^{n} d_{i}^{2}=10 n,
$$

then the following equations hold

$$
\begin{gathered}
\sum_{i=1}^{4}(i-2) n_{i}=-n_{1}+n_{3}+2 n_{4}=0, \\
\sum_{i=1}^{4}\left(i^{2}-i-3\right) n_{i}=-3 n_{1}-n_{2}+3 n_{3}+9 n_{4}=0 .
\end{gathered}
$$

Combining Equations (3.1), (3.2) and (3.3), we arrive at

$$
n_{3}+3 n_{4}=n .
$$

So by Theorem 2.12, two graphs $\mathcal{L}(\mathcal{G})$ and $\mathcal{L}\left(S G_{n}\right)$ are $A$-cospectral (where $A$ is the adjacency matrix), then $t(\mathcal{L}(\mathcal{G}))=t\left(\mathcal{L}\left(S G_{n}\right)\right)$. Therefore, two cases occur:

Case 1. If $t(\mathcal{G})=\alpha>0$, then by Lemma 2.9 we have $n_{3}+4 n_{4}+\alpha=n$ and Equation (3.4) yields $n_{4}=-\alpha$, a contradiction.

Case 2. If $t(\mathcal{G})=0$, then by Lemma 2.9 we have $n_{3}+4 n_{4}=n$. Also Equations (3.2), (3.3), and (3.4) imply that $n_{4}=n_{2}=0, n_{1}=n_{3}=n$. Furthermore, the order of each cycle of $\mathcal{G}$ is at most $n$.

In particular, if $\mathcal{G}$ has a cycle of order $k(3<k<n)$, then $\mathcal{G}$ has a proper subgraph $\mathcal{G}^{\prime} \cong S G_{k}$ because each cycle vertex has degree 3 . Therefore, the interlacing theorem implies that $\nu_{1}(\mathcal{G})>\nu_{1}\left(\mathcal{G}^{\prime}\right)$ which is a contradiction with Corollary 3.3. Thus $k=n$, and so $\mathcal{G}$ must be isomorphic to $S G_{n}$.

TheOREm 3.5. Let $n$ be an odd number. Then the Sun graph, $S G_{n}$, is determined by its $Q$-spectrum.

Proof. Suppose that $\mathcal{G}$ is $Q$-cospectral with $S G_{n}$. By Theorem 2.7 we have $\operatorname{det}(Q(\mathcal{G}))=\operatorname{det}\left(Q\left(S G_{n}\right)\right)=4$. Therefore $\mathcal{G}$ is an odd unicyclic graph. Suppose that $n=3$, it is easy to check that, $\mathcal{G}$ must be one of the graphs in Fig. 2. Moreover, Theorem 2.12 implies that $t(\mathcal{L}(\mathcal{G}))=t\left(\mathcal{L}\left(S G_{n}\right)\right)=4$, in which $t(\mathcal{G})$ denotes the number of triangles of $\mathcal{G}$. Obviously, no graph in Fig. 2 has this property.

By Theorem 3.4 and the fact that $\mathcal{G}$ is an odd unicyclic graph, we have $\mathcal{G} \cong S G_{n}$, for odd $n \geq 5$. This completes the proof.

Next, suppose that $n$ is an even number. Since $Q\left(S G_{n}\right)=L\left(S G_{n}\right)$, we have $\operatorname{rank}(Q(\mathcal{G}))=\operatorname{rank}\left(Q\left(S G_{n}\right)\right)=2 n-1$. Hence, by Theorem $2.11, \mathcal{G}$ has one bipartite component. 

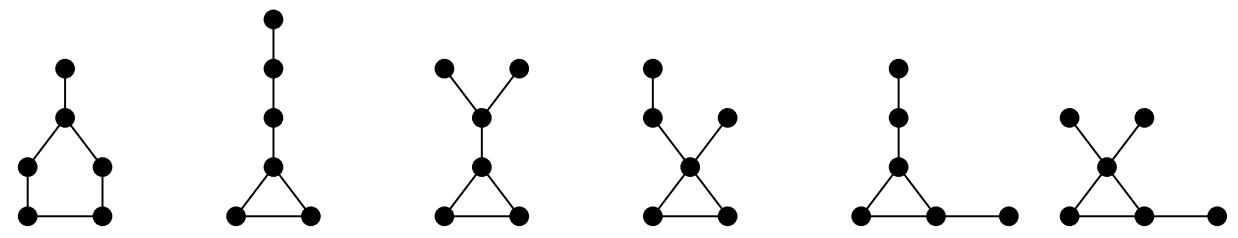

Fig. 2.

If $\mathcal{G}$ is a graph with no vertex of degree 0 , then by Theorem $3.4, \mathcal{G} \cong S G_{n}$. Therefore, suppose that $\mathcal{G}$ has an isolated vertex, and $\mathcal{G}_{1}, \ldots, \mathcal{G}_{c}$ are non-bipartite connected components of $\mathcal{G}$ where $c \geq 1$. Also without loss of generality we may assume that $\mathcal{G}_{1}, \ldots, \mathcal{G}_{c-1}$ are odd unicyclic graphs, and $\mathcal{G}_{c}$ is bicyclic.

Similar to the proof of the Theorem 3.4, we have $n_{3}+3 n_{4}=n-1$. Also Lemma 2.9 implies that $n=t(\mathcal{G})+n_{3}+4 n_{4}$. By combining these equations, two following cases occur:

Case 1. If $t(\mathcal{G})=0$, then $n_{4}=1, n_{2}=6, n_{1}=n_{3}=n-4$. Furthermore, the graph $H$ which is shown in Fig. 3 has $3+\sqrt{5}$ as its largest $Q$-eigenvalue. Hence, it can not be a subgraph of $\mathcal{G}$, because of the interlacing theorem. Therefore, the vertex of degree 4 has at least two neighbors of degree 1 . We get a contradiction by Lemma 2.8 and Corollary 3.2 .

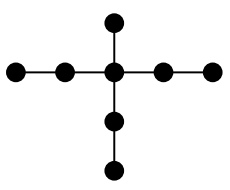

Fig. 3. The graph $H$

Case 2. If $t(\mathcal{G})=1$, then $n_{4}=0, n_{3}=n-1, n_{2}=3$, and $n_{1}=n-3$.

Noting that the adjacency spectrum of $C_{n}$ is $\lambda_{i}=2 \cos \frac{2 \pi i}{n}$, for $i=1, \ldots, n$. But in fact, these numbers are not all distinct. By Lemma 3.1, the $Q$-spectrum of $S G_{n}$ is in the form of

$$
(3+\sqrt{5})^{1},\left(2+\lambda_{i}+\sqrt{1+\left(1+\lambda_{i}\right)^{2}}\right)^{2}, 2^{1},(3-\sqrt{5})^{1},\left(2+\lambda_{i}-\sqrt{1+\left(1+\lambda_{i}\right)^{2}}\right)^{2}, 0^{1},
$$

in which $\lambda_{i}=\cos \frac{2 \pi i}{n}$ and $i=1, \ldots, \frac{n-2}{2}$.

Hence, the product of the $Q$-eigenvalues of $S G_{n}$ with multiplicity 2 equals $\gamma=$ $\frac{n^{2}}{4}$, because $\zeta_{2 n-1}\left(S G_{n}\right)=2 n^{2}=\prod_{\nu_{i} \neq 0} \nu_{i}\left(S G_{n}\right)=8 \gamma$.

Let $H$ be a proper subgraph of $\mathcal{G}$, which is obtained by deleting an edge from $\mathcal{G}_{c}$ such that all its non-bipartite connected components are odd unicyclic graphs. 
Obviously, $H$ is a $T U$-subgraph of $\mathcal{G}$ with $2 n-1$ edges, containing $c$ odd unicyclic connected components and one isolated vertex. By Theorem 2.4 and the interlacing theorem, we have

$$
W(H)=4^{c}=\prod_{i=1}^{2 n-1} \nu_{i}(H)=\sqrt{\gamma} \alpha .
$$

Now using Theorem 2.3, if $\alpha$ is a rational number then $\alpha$ is an integer number, because the characteristic polynomial of the adjacency matrix of a graph is a monic polynomial with integer coefficients. Therefore, there exists a positive integer $\beta$, such that $n=2^{\beta}$.

REMARK 3.6. Suppose that $\mathcal{G}$ has a cycle whose vertices have degree 3 , and any two cycle vertices have no common neighbor outside of the cycle. Thus using Corollary 3.3 and interlacing theorem, $\mathcal{G}$ must be isomorphic to $S G_{n}$.

Lemma 3.7. Let $\mathcal{G}$ be $Q$-cospectral with $S G_{2^{\beta}}$, for positive integer $\beta$. Suppose that $k$ is an odd number and $v$ is a pendent vertex. Then $\mathcal{G}$ has no connected component $H$ isomorphic to $S G_{k}-v$.

Proof. By applying the interlacing theorem and Lemma $3.1, H$ has $2+\cos \frac{2 \pi}{k}+$ $\sqrt{1+\left(1+\cos \frac{2 \pi}{k}\right)^{2}}$ as its $Q$-eigenvalue. We show that $Q\left(S G_{n}\right)$ does not have such eigenvalue. Assume otherwise, so there exists some $i\left(1 \leq i \leq n=2^{\beta}\right)$ such that

$$
2+\cos \frac{2 \pi}{k}+\sqrt{1+\left(1+\cos \frac{2 \pi}{k}\right)^{2}}=2+\cos \frac{2 \pi i}{n} \pm \sqrt{1+\left(1+\cos \frac{2 \pi i}{n}\right)^{2}},
$$

which implies that $\cos \frac{2 \pi}{k}=\cos \frac{2 \pi i}{n}$ for some $i$. One may see that it is impossible for an odd $k$ and $n=2^{\beta}$, and the result follows.

By Remark 3.6, each non-bipartite connected component of $\mathcal{G}$ must contain at least one vertex of degree 2. It follows that $c \leq n_{2}=3$, where $c$ is the number of non-bipartite connected components of $\mathcal{G}$.

If $c=3$, then $\mathcal{G}$ does not have a vertex of degree 3 outside of any cycle, by Lemma 2.8. So $\mathcal{G}$ has an odd unicyclic connected component $\mathcal{G}_{1} \cong S G_{k}-v$, in which $v$ is a pendent vertex and $k$ is an odd number. This is a contradiction by Lemma 3.7, and consequently $c \leq 2$.

Now suppose $\mathcal{G}_{c}$ (the bicyclic component of $\mathcal{G}$ ) is $\theta_{p, q, r}$ with trees attached. Also let $\kappa$ be the number of edges such as $e \in E\left(\mathcal{G}_{c}\right)$, for which the graph $\mathcal{G}_{c}-e$ is an odd unicyclic graph.

If $c=2$, then $\zeta_{2 n-1}=2 n^{2}=4^{2} \kappa$, and $\kappa \leq n-1$. Therefore, $n^{2} \leq 8(n-1)$ and it implies that $n=4$, because $n=2^{\beta}$. On the other hand, for $c=1$, similar to the 
above we have $\zeta_{2 n-1}=2 n^{2}=4 \kappa$, where $\kappa \leq n+2$. Hence the only possible case is $n=4$.

But for $n=4$, by our assumption the graphs in Fig. 4 are the possible graphs. By computing the quantity $\zeta_{2 n-1}$, these graphs are not desired.
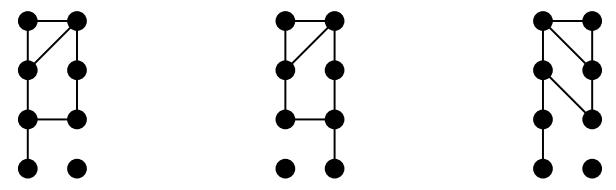

Fig. 4.

Also if $\mathcal{G}_{c}$ is $\gamma_{p, q, r}$ with trees attached and $c=2$, then using Lemma 2.8 and Corollary $3.2, \mathcal{G}$ has no vertex of degree 3 outside of any cycle. So we may suppose that $\mathcal{G}$ has an odd unicyclic connected component $\mathcal{G}_{1} \cong S G_{k}-v$, where $v$ is a pendent vertex and $k$ is an odd number. This is a contradiction by Lemma 3.7. Therefore, $c=1$ and by Equation (3.5), we have $4=\frac{n}{2} \alpha$ and it follows that $n \in\{4,8\}$.

Evidently, for $n=4, \mathcal{G}$ must be isomorphic to the graph which is shown in Fig. 5 . We know, that is a contradiction with $t(\mathcal{G})=1$.

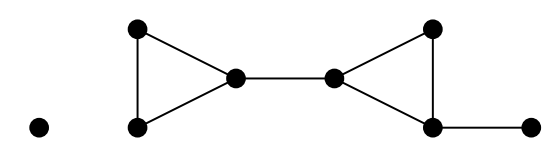

Fig. 5.

Finally, for $n=8$, we have two choices for deleting an edge from $\mathcal{G}$ to obtain a $T U$-subgraph with $2 n-1$ edges. One may either choose the edge $e$ from the path $P_{r}$ where $0 \leq r \leq 4$ or choose the edge $e$ from cycles $C_{p}$ or $C_{q}$ where $p+q \leq 10$. Therefore, we have $\zeta_{2 n-1}(\mathcal{G})=2 n^{2}=128 \leq 4^{2}(r+1)+4(p+q) \leq 120$, a contradiction. Hence, $\mathcal{G}$ can not contain an isolated vertex.

Accordingly we may summarize our results as follows.

TheOREM 3.8. The Sun graph is determined by its $Q$-spectrum.

Acknowledgment. The authors thank the referee for his or her constructive comments. 


\section{REFERENCES}

[1] R. Boulet. Spectral characterizations of sun graphs and broken sun graphs. Discrete Math. Theor. Comput. Sci., 11:149-160, 2009.

[2] D. Cvetković, M. Doob, and H. Sachs. Spectra of Graphs - Theory and Applications, Third edition. Johann Ambrosius Barth. Heidelberg, 1995.

[3] D. Cvetković, P. Rowlinson, and S. Simić. Eigenvalue bounds for the signless Laplacian. Publ. Inst. Math. (Beograd) (N.S.), 81(95):11-27, 2007.

[4] D. Cvetković, P. Rowlinson, and S. Simić. Signless Laplacians of finite graphs. Linear Algebra Appl., 423:155-171, 2007.

[5] Y. Fan and D. Yang. The signless Laplacian spectral radius of graphs with given number of pendant vertices. Graphs Combin., 25:291-298, 2009.

[6] R. Grone and R. Merris. The Laplacian spectrum of a graph. II. SIAM J. Discrete Math., 7:221-229, 1994.

[7] W. H. Haemers, X. Liu, and Y. Zhang. Spectral characterizations of lollipop graphs. Linear Algebra Appl., 428:2415-2423, 2008.

[8] M. Jooyandeh, D. Kiani, and M. Mirzakhah. Incidence energy of a graph. MATCH Commun. Math. Comput. Chem., 62:561-572, 2009.

[9] R. Merris. Laplacian matrices of graphs: a survey. Linear Algebra Appl., 197/198:143-176, 1994.

[10] G. R. Omidi. On a signless Laplacian spectral characterization of $T$-shape trees. Linear Algebra Appl., 431:1607-1615, 2009.

[11] E. van Dam and W. H. Haemers. Which graphs are determined by their spectrum? Linear Algebra Appl., 373:241-272, 2003. 